\title{
A Method of Face Detection Based On Improved YCBCR Skin Color Model
}

\author{
Fan Jihui ${ }^{1,}$, , Wang Hongxing ${ }^{2, b}$ \\ ${ }^{1}$ School of Energy Science and Engineering, Henan Polytechnic University, Jiaozuo 454003, China

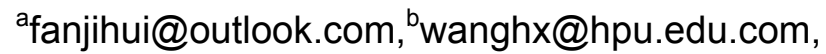

\begin{abstract}
Keywords: Face detection Skin color Clustering feature Color space Mark image
\end{abstract}
\begin{abstract}
Face detection is a key technology in the information processing field of face recognition. The paper proposes a new face detection method to detect the human face. According to the clustering characteristics of $\mathrm{Cb}$ and $\mathrm{Cr}$ to detect the skin-color, then the image is labeled. The paper summarizes and presents a localization ears algorithm of human face. It also presents a method to locate the human chin based on the center line of pixel gray [CLPG]. The two methods can remove the interference of the ear and neck region. Experimental results show that the method can detect face quickly and accurately in an image and provide a certain value for the study of face recognition.
\end{abstract}

\section{Introduction}

Vision is the main means for human to get information from the nature world. According to the statistics, visual information is about $60 \%$, auditory information is about $20 \%$, other way adds up to about $20 \%^{[1]}$. This shows the importance of visual information to mankind. In color images, skin color information is used to detect human face, which can provide more accurate search range and face positioning. Therefore, the skin color has important research and great application value.

Compared with processing gray level information, face skin color has some advantages, like that it is not sensitive to light, attitude change, and contains a wealth of facial information. It is widely used in skin color feature extraction, face detection, face recognition, and some other research directions. Among them, skin detection based on $\mathrm{YCbCr}$ color space is the most widely used method ${ }^{[2]}$. In $\mathrm{YCbCr}$ space, the distribution of $\mathrm{Cb}$ and $\mathrm{Cr}$ components of facial color tends to be consistent. It can effectively remove the illumination brightness effect of $\mathrm{Y}$, and it also has a good clustering feature ${ }^{[3]}$. Based on the above characteristics, many researchers have carried out the study of human face detection. For example, Wu Yaoling, from the University of Electronic Science and technology, uses template matching and clustering characteristics to detect skin color in the YCbCr space. That way can effectively detect the position of the human face and calibrate its position in a complex background ${ }^{[4]}$. However, his algorithm is complex, and it needs to be improved in positioning accuracy; Wang Lijuan, from Sichuan University, uses a fast face detection algorithm based on skin color ${ }^{[5]}$. Although the detection time is shortened, the detection rate and the accuracy are insufficient.

This paper uses MATLAB 7 as the experimental platform. Firstly, do some in-depth study about $\mathrm{YCbCr}$ space and the clustering characteristic of $\mathrm{Cb}$ and $\mathrm{Cr}$ components; Secondly, build the skin color model for color separation and remove interference of the small area; Thirdly, select the candidate faces; Finally, the paper presents a localization ears algorithm to remove ear region, and anther method based on CLPG is proposed to remove the human chin's interference.

\section{Face detection method}

Compared with other features, skin-color has the following characteristics ${ }^{[6]}$ : it is not affected by posture and size; regardless of whether the face is spinning and the expression changes, skin color are available; it is not sensitive to direction. Although the light source will affect the skin-color, some auxiliary method can be used to correct. The method in the paper is a face detection based on static image, belonging to the feature-based method.

The algorithm flow of this paper is simplified as shown in Fig.1: 


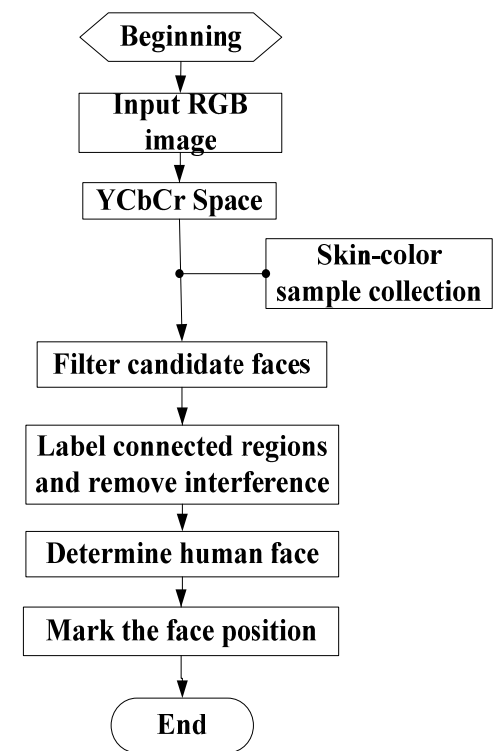

Fig. 1 Flow chart

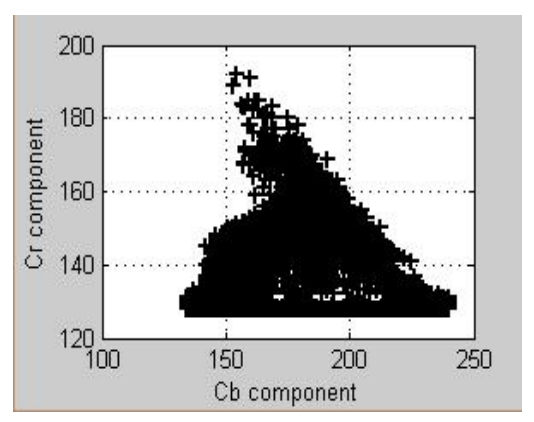

Fig.2 The clustering characteristic of $\mathrm{Cb}, \mathrm{Cr}$

\section{Skin color modeling}

\subsection{YCbCr color space}

In the JPEG standard, the image is converted from the RGB color space to the Luminance -chrominance spaces. The converted space is called $\mathrm{YCbCr}$ color space. Among it, $\mathrm{Y}$ is the luminance, $\mathrm{Cb}$ is blue component, $\mathrm{Cr}$ is red component.

Conversion formula of RGB color space to $\mathrm{YCbCr}$ color space is shown as formula (1):

$$
\left[\begin{array}{c}
Y \\
C b \\
C r
\end{array}\right]=\left[\begin{array}{ccc}
0.257 & 0.504 & 0.098 \\
-0.148 & -0.219 & 0.439 \\
0.439 & -0.368 & -0.071
\end{array}\right]\left[\begin{array}{l}
R \\
G \\
B
\end{array}\right]+\left[\begin{array}{c}
16 \\
128 \\
128
\end{array}\right]
$$

The clustering properties of $\mathrm{Cr}, \mathrm{Cb}$ in $\mathrm{YCbCr}$ space are shown as Fig.2.

\section{2 skin-color model}

In order to establish the skin-color model, $100144 * 192$ pixels color images are cut into $63 * 85$ pixels which contain mainly the face. After going on a low-pass filter, they will be conversed from the RGB space to YCbCr space. According to the statistics , obtain $\mathrm{Cb} \in(128,240), \mathrm{Cr} \in(128,166)$.

The values of $\mathrm{Cb}$ and $\mathrm{Cr}$ are brought into the formula (2):

$U(i, j)=\left\{\begin{array}{cc}1, & \left(C b_{-} \min \leq C b(i, j) \leq C b_{-} \max , C r_{-} \min \leq C r(i, j) \leq C r_{-} \max \right) ; \\ o, & \text { other; }\end{array}\right.$

In the formula, $\mathrm{U}$ is a logical matrix. The white means skin-color regions, as shown in Fig.3 (b).

\section{Precise positioning face region}

\subsection{Select candidate face}

After going on mathematical morphology processing, the paper adopts the method of area ratio to eliminate these interference of small connected regions. Human face aspect ratio is between 1.1 and 1.6. Due to the interference of neck, the range is expanded to between 1.0 and 2.6. Filtered face region in experiment is shown in Fig.3 (c).

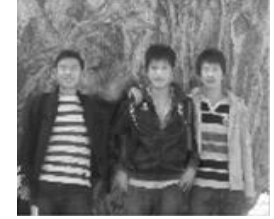

(a)Color image

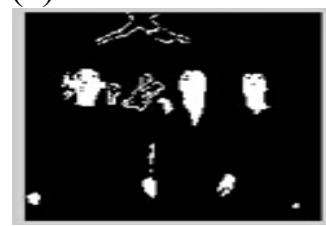

(b) Skin-color separation

Fig.3 Skin color separation

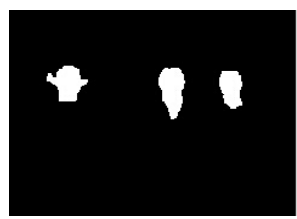

(c) Selected face 


\subsection{Ear root positioning}

In order to eliminate the ear region of the candidate's face, this paper proposes an ear root positioning method. It is shown in Fig.4. Among the connected region, the 'width' is width; col_min and col_max are respectively the min and max column; L_ear and R_ear are respectively the column of left and right ear roots; col_centre is the central line.

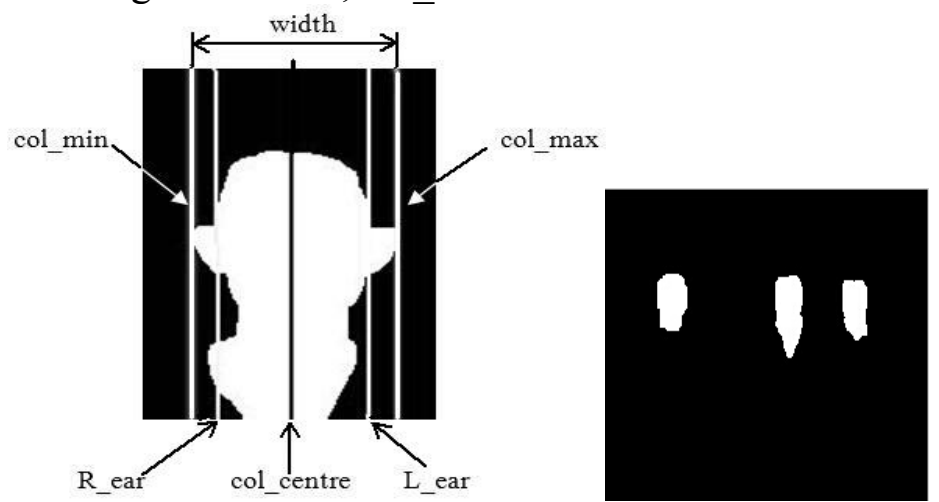

Fig.4 The position of the ears
Fig. 5 Eliminate ear interference

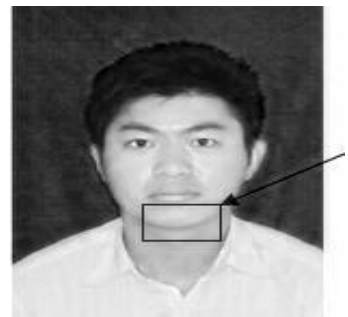

Fig.6 The gray change of the chin

Methods are as follows: (1) Mark the connected regions that contains the face;

(2) If Fig.4 is the $\mathrm{N}$-th $(\mathrm{N}=1,2, \ldots)$ connected region, the gray value of the connected region is $\mathrm{N}$;

(3) Searching image by column, find out the max column col_max and the min column col_min of the n-th connected region. And then, according to the formula (4), the col_centre of the connected region is calculated out:

$$
\text { col_centre }=\mathrm{col} \_\min +\left(\mathrm{col} \_\max -\mathrm{col} \_\min +1\right) / 2
$$

(4) Let Ln is the pixels' number of n-th column in the n-th connected region. Statistics the every column pixel number of the connected regions. Among it, the column value of $\mathrm{n}$ is from col_min to col_centre. Respectively, they are recorded as $\mathrm{L}_{1}, \mathrm{~L}_{2}, \ldots, \mathrm{L}_{\mathrm{k}}, \mathrm{k}=$ col_centre-col_min +1 ;

(5) Judgment the formula (5), and find out the all $\mathrm{L}_{\mathrm{i}}(\mathrm{I} \in(1, \mathrm{k}-1))$ that the proportion is less than the threshold. And then record all $\mathrm{L}_{\mathrm{i}}$ corresponding to the column $\mathrm{C}_{\mathrm{i}}, \mathrm{C}_{1}, \mathrm{C}_{2}, \ldots, \mathrm{C}_{\mathrm{i}}$ form a matrix. The matrix is recorded as $A=[C 1, C 2, \ldots]$. In matrix $A$, the max $C_{-} \max$ is calculated. The $C_{-} \max$ is the right ear root location $\mathrm{R}$ _ear. Here, according to the ratio of ear length account for face and a large number of experimental statistics, the range of thresh is between 0.20 and 0.30 .

$$
0.20 \leq \frac{L_{1}}{L_{k}}, \frac{L_{2}}{L_{k}}, \ldots, \frac{L_{k-1}}{L_{k}} \leq 0.30
$$

(6) Using the principle of (4) and (5) to calculate the ear root location L_ear of the other side face;

(7) For the connected region, if its column coordinates are not in the range [L_ear R_ear], then its gray value is set to 0 . Thereby, the interference of the ears regions is eliminated, as shown in Fig.5. 4.3 Cycle detection method based on CLPG

The gray has an obvious change between the chin and neck in the image, as shown in Fig.6. Therefore, this paper proposes a cycle detection method based on CLPG to remove interference of the neck. The specific methods are as following:

(1) Remove the ears' interference.

(2) Calculate the image-processed vertical center line col_centre and the left-most pixel column coordinate $\mathrm{L}$, the right-most pixel column coordinate $\mathrm{R}$, the row coordinate $\mathrm{H}$ of the top pixel and the column coordinate $\mathrm{M}$ of the bottom pixel. According to the formula (6), the face's width is obtained.

$$
\text { width }=R-L+1
$$

(3) According to the ratio $\in\left[\begin{array}{ll}1.1 & 1.6\end{array}\right]$ of face length and width, using the formula (7), the lower boundary $\min$ row $Q \_\min ($ when $Q$ it takes the min 1.0) and the maximum row Q_max (when Q takes the max 1.6) of the face region are anticipated.

$$
Q=\text { width } \times \text { ratio }+H
$$


(4) Find out all the pixels' coordinates that their gray value are 1 (if it is the $\mathrm{N}$-th block connected region, the gray value is $\mathrm{N}$ ) on the center line of the connected regions. And they will be recorded as (H, col_centre),..., (M, col_centre); According to the coordinates above to take out the pixels' gray values of the corresponding gray image. The gray values are placed in a matrix B.

(5) Calculate the min V_min of the matrix B and its abscissa $\mathrm{V}$. $\mathrm{h}$ in the image. Then to get the

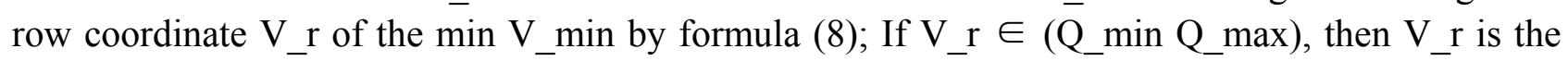
location of chin, otherwise $V_{-}$r will be not. In this case, the min V_min in the matrix B will be set to 255, and the V_min and its coordinate $\mathrm{V} \_\mathrm{h}, \mathrm{V} \_\mathrm{r}$ will be recalculated.

$$
V_{-} r=\bar{V}_{-} h+H-1
$$

(6) Remove those pixels of the connected region that the row coordinates are more than $\mathrm{V} \_\mathrm{r}$ and the gray value is 1 ; if there are multiple connected regions, the interference in the $n$-th connected area should be removed according to its corresponding V_r.

Use the method above, the experimental results is got, as shown in Fig.7 (a), (b).

In this paper, the accuracy of face location is shown in Fig.8 (c), (d), (e):

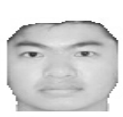

(a) Single face

Fig.7 Remove interference under the chin

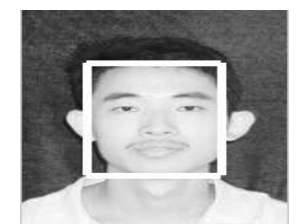

(c) Single face

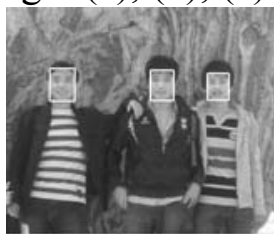

(d) The model (front)

Fig.8 The detection results of this paper

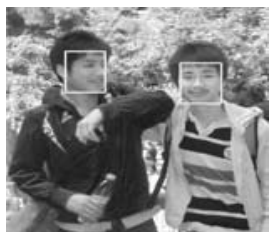

(e) The model (flank)

\section{Conclusion}

A lot of experiments prove that compared to the traditional Gauss skin color model, this method can accurately locate the face. Even if the face has a certain degree of tilt when it is taken, it can work. The method can provide a certain basis, reference and research value for the face recognition in the latter. Use above method to detect the face on 270 photos, the detection rate is shown in table 1.

Table 1 Face detection rate comparison

\begin{tabular}{|c|c|c|}
\hline Model & Single face & Multi face \\
\hline Gauss skin color model & $84.6 \%$ & $71.0 \%$ \\
\hline The model of this paper & $97.4 \%$ & $94.7 \%$ \\
\hline
\end{tabular}

\section{Acknowledgments}

This work was financially supported by the Key Scientific Research Project of Henan Province (15A510024).

\section{References}

[1] Hou B W, Yang G S. Face detection and identification [J]. Journal of Minzu University of China (Natural Science Edition), 2013, 22 (4): 57-62.

[2] Shi D C, Cai F. Facial Expression Recognition Based on Gabor Wavelet Phase Features[C]. Proc of the 7 th International Conference on Image and Graphics. 2013: 520-523.

[3] VERMA A, RAJ SA, MIDYA A, CHAKRABORTY J. Face detection using skin color modeling and geometric feature. Informatics, Electronics \& Vision (ICIEV), 2014 International Conference on Dhaka, 2014: 1-06

[4] Wu Y L. The design and implementation of face detection algorithm based on YCrCb color space [D]. Xi'an: University of Electronic Science and Technology, 2013 
[5] Banerjee P K, Datta A K. An illumination tolerant class specific 2D subspace based face recognition technique using optimum correlation filter [J]. Optik-International Journal for Light and Electron Optics, 2013, 124(17): 3173-3179

[6] Ma X H, Tan Y Q. Based on the identification of sparse embedding of face recognition algorithm [J]. Automation of the Journal, 2014, 40 (1): 73-82 